\title{
ESTRUTURA FITOSSOCIOLÓGICA, ESTOQUES DE VOLUME, BIOMASSA, CARBONO E DIÓXIDO DE CARBONO EM FLORESTA ESTACIONAL SEMIDECIDUAL ${ }^{1}$
}

\author{
Agostinho Lopes de Souza ${ }^{2}$, Aline Boina ${ }^{3}$, Carlos Pedro Boechat Soares², Benedito Rocha Vital², Ricardo \\ de Oliveira Gaspar ${ }^{3}$ e Jacinto Moreira de Lana ${ }^{4}$

\begin{abstract}
RESUMO - O objetivo deste estudo foi analisar o estoque e o crescimento em volume, biomassa, carbono e dióxido de carbono em duas áreas de Floresta Estacional Semidecidual Submontana em estágios médio e médio/avançado de sucessão da vegetação secundária. Foram utilizados dados de inventários de parcelas permanentes medidas em 2002 e 2007. Para determinar as densidades básicas da madeira e da casca das árvores, foram selecionadas espécies que apresentavam maiores porcentuais em volume e, no mínimo, cinco indivíduos. Na área em estágio médio de sucessão da vegetação secundária, os crescimentos em volume, biomassa, carbono

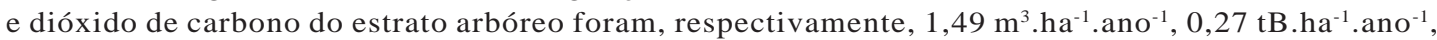
10,13 tC.ha- ${ }^{-1}$.ano ${ }^{-1}$ e 0,50 tCO $_{2} \cdot$ ha $^{-1} \cdot$ ano $^{-1}$. A área em estágio médio avançado de sucessão apresentou

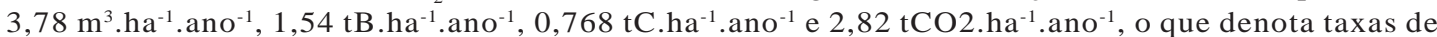
crescimento relativo do estoque de dióxido de carbono de $0,37 \%$ na área em estágio médio de sucessão e 1,05\% na área em estágio médio avançado. As espécies com maiores estoques e crescimentos na área em estágio médio foram de Mabea fistulifera, Apuleia leiocarpa, Pouteria torta, Brosimum guianense e Pseudopiptadenia contorta e, na área em estágio médio avançado de sucessão, foram Pseudopiptadenia contorta, Mabea fistulifera, Apuleia leiocarpa, Brosimum guianense, Tapirira guianensis e Cupania oblongifolia.
\end{abstract}

Palavras-chave: Crescimento, Sucessão secundária e Sequestro de carbono.

\section{PHYTOSOCIOLOGICAL STRUCTURE, VOLUME STOCKS, BIOMASS, CARBON AND CARBON DIOXIDE IN SEASONAL SEMIDECIDUOS FOREST}

\begin{abstract}
The objective of this work was to analyse the stock and volume growth, biomass, carbon and carbon dioxide in two areas of Semideciduos Submontane Seasonal Forest in intermediate and intermediateadvanced successional stages of the secondary vegetation. It was used data from surveys of the permanent plots measured in 2002 and 2007. To determine the basic density of the wood and bark of the trees, the species with the greatest percentage in volume with at least five (5) individuals were selected. In the areas at intermediate succession stage, growths in volume, biomass, carbon and carbon dioxide of the tree strata were $1.49 \mathrm{~m}^{3}$. ha-

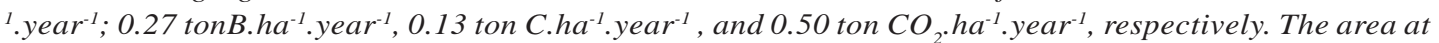
intermediate-advanced succession stage showed growths of $3.78 \mathrm{~m}^{3} . \mathrm{ha}^{-1}$.year $\mathrm{r}^{-1} ; 1.54$ tonB. ha ${ }^{-1}$.year ${ }^{-1}, 0.77$ ton C.ha ${ }^{-1}$ year ${ }^{-1}$, and 2.82 ton $\mathrm{CO}_{2}$.ha ${ }^{-1}$.year-1, which show a relative increase of carbon dioxide stocks rate of $0.37 \%$ in the intermediate succession stage and of $1.05 \%$ in the intermediate-advanced stage. The populations with greater importance values, stocks and growths in the intermediate stage area were of Mabea fistulifera, Apuleia leiocarpa, Pouteria torta, Brosimum guianense and Pseudopiptadenia contorta, and in the intermediateadvanced area they were of Pseudopiptadenia contorta, Mabea fistulifera, Apuleia leiocarpa, Brosimum guianense, Tapirira guianense and Cupania oblongifolia.
\end{abstract}

Keywords: Growth, Secundary succession and Carbon sequestration..

\footnotetext{
${ }^{1}$ Recebido em 29.09.2010 e aceito para publicação em 15.11.2011.

2² Departamento de Engenharia Florestal da Universidade Federal de Viçosa, UFV, Brasil. E-mail:<alsouza@ufv.br>, csoares@ufv.br e <bvital@ufv.br>.

${ }^{3}$ Programa de Pós-Graduação em Ciencia Florestal pela Universidade Federal de Viçosa, UFV, Brasil. Email:<alineboina@yahoo.com.br> e <ricogaspar.floresta@yahoo.com.br>

${ }^{4}$ Celulose Nipo Brasileira, CENIBRA, Brasil. E-mail: <jacinto.lana@cenibra.com.br>.
} 


\section{INTRODUÇÃO}

No Brasil, as florestas naturais e as implantadas são um dos principais pilares de edificação do desenvolvimento nacional sustentável. A cobertura vegetal natural remanescente dos biomas Cerrado, Caatinga, Pantanal, Amazônia e Floresta Atlântica, além de estocar e capturar Carbono (MCT, 2006), são depositários de elevada diversidade biológica (MYERS et al., 2000; VIEIRA et al., 2008) e, simultaneamente, prestam serviços ambientais e proporcionam produtos de diversas cadeias produtivas (PASSOS; BRAZ, 2004).

A importância das florestas no equilíbrio do estoque de carbono global é inquestionável (CHAVE et al., 2005; MCT, 2006; VIEIRA et al., 2008), visto que armazenam em suas árvores e no solo quantidades de carbono superiores às existentes na atmosfera (LAL et al., 1995; SILVA; MACHADO, 2000; FRA, 2010).

As florestas são fontes e sumidouros de carbono atmosférico. Emitem $\mathrm{CO}_{2}$ devido a respiração, queima e decomposição da biomassa e, no entanto, são sumidouros devido a fotossíntese e crescimento da biomassa (HOEN; SOLBERG 1994; FOODY et al., 1996). Nas florestas naturais tropicais predominam indivíduos arbóreos de diferentes espécies, idades e tamanhos. A produção de biomassa e carbono está concentrada no componente arbóreo e associada com a composição de espécies (MULLER-LANDAU, 2004), tamanho populacional, tamanho das árvores (diâmetro e altura), idade, densidade básica da madeira (SWAINE; WHITMORE, 1988; BARNETT; JERONIMIDIS, 2003) e estágio de sucessão da floresta (WATZLAWICK et al., 2002).

O objetivo deste estudo foi analisar os estoques de volume, biomassa, carbono e dióxido de carbono, das principais espécies, em duas áreas de reservas protegidas em processos de recuperação ambiental inserida no projeto de base florestal da CENIBRA, região fitoecológica da Floresta Estacional Semidecidual Submontana, bioma Mata Atlântica, Vale do Rio Doce, MG.

\section{MATERIAL E MÉTODOS}

\section{1. Área de Estudo}

As áreas de estudo, doravante denominadas Mata 1 ( $19^{\circ} 30^{\prime} 24^{\prime \prime}$ S; $42^{\circ} 25^{\prime} 50^{\prime \prime}$ ' W) e Mata 2

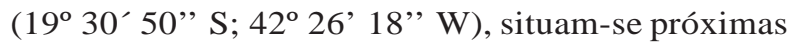

e às margens da Lagoa Silvana e pertencem aos Municípios de Caratinga e Bom Jesus do Galho, Vale do Rio Doce, MG. A tipologia vegetal original inclui-se na região fitoecológica da Floresta Estacional Semidecidual Submontana (VELOSO et al., 1991; IBGE, 2004). O clima é tropical úmido (Aw), com inverno seco e verão chuvoso; temperatura máxima média de $32,2{ }^{\circ} \mathrm{C}$ e mínima média de $19,4{ }^{\circ} \mathrm{C}$; umidade relativa média de 59,8\%; precipitação pluviométrica média mensal de 106,6 mm; e evapotranspiração potencial anual entre 950 e $1.200 \mathrm{~mm}$.

A área pertence à empresa Celulose Nipo Brasileira S.A. - CENIBRA e tem 302 ha de mata nativa, a qual está preservada há aproximadamente 20 anos, sendo dividida em duas partes: Mata 1, com 272 ha; e Mata 2, com 30 ha.

\subsection{Amostragem e Coleta de Dados}

A dinâmica de sucessão das áreas de estudo foi monitorada por meio de parcelas permanentes de $500 \mathrm{~m}^{2}$ (0,05 ha) cada, instaladas e inventariadas em 2002 e 2007. Foram lançadas seis parcelas permanentes na Mata 1 e 16 na Mata 2, perfazendo $3.000 \mathrm{~m}^{2}$ (0,30 ha) e $8.000 \mathrm{~m}^{2}$ (0,80 ha) de área amostral, respectivamente.

Cada árvore do estrato arbóreo (DAP $\geq 5,0 \mathrm{~cm}$ ) foi identificada, quando possível, em nível de espécie e teve o DAP e as alturas total (Ht) e comercial (Hc) mensuradas, bem como foi realizada a sua localização dentro da parcela através de coordenadas x e y.

Espécies foram selecionadas para a amostragem do tronco para a determinação das densidades básicas e aparentes da madeira e da casca. Dados do inventário realizado em 2002 foram utilizados, sendo selecionadas as espécies que apresentavam, à época, maiores porcentuais em volume e, no mínimo, cinco indivíduos.

Para cada espécie selecionada foram escolhidos aleatoriamente, nas parcelas de inventário, cinco indivíduos do estrato arbóreo para coletas de madeira e casca, pelo método do trado.

\subsection{Análises dos Dados}

Os dados do inventário das parcelas permanentes coletados em 2002 e 2007 foram analisados para obtenção do índice de diversidade de Shannon-Weaver (BROWER e ZAR, 1984) e dos parâmetros fitossociológicos (MUELLER-DOMBOIS; ELLEMBERG, 1974). 
A determinação da densidade básica seguiu a metodologia da ABNT (1997) e de Vital (1984).

Os volumes total, de fuste e de copa, com e sem casca, por espécie, foram estimados mediante o emprego de equações de volumes de árvores individuais ajustadas para Mata Secundária (SOARES et al., 2006), sendo os volumes de casca do fuste (VFc), de galho com casca (VGcc) e de casca dos galhos (VGc) obtidos pelas seguintes expressões (BOINA, 2008):

$v f c_{i j}=v f c c_{i j}-v f s c_{i j} ; v g c c_{i j}=v t c c_{i j}-v f c c_{i j}$;

$v g c_{i j}=v g c c_{i j} \cdot F c a s c a ; \mathrm{e}$

Fcasca $=\sum_{i=1}^{n} v f_{i} / \sum_{i=1}^{n} v f c c_{i}$

em que $v f c_{i j}=$ volume de casca da $j$-ésima árvore da i-ésima espécie, em $\mathrm{m}^{3} ; v f c c_{i j}=$ volume do fuste com casca da $j$-ésima árvore da i-ésima espécie, em $\mathrm{m}^{3} ; v f s c_{i j}=$ volume do fuste sem casca da $j$-ésima árvore da i-ésima espécie, em $\mathrm{m}^{3} ; v g c c_{i j}=$ volume de galho com casca da j-ésima árvore da i-ésima espécie, em $\mathrm{m}^{3} ; v t c c_{i j}=$ volume total com casca da $j$-ésima árvore da i-ésima espécie, em $\mathrm{m}^{3} ; v g c_{i j}=$ volume de casca do galho da $j$-ésima árvore da i-ésima espécie, em $\mathrm{m}^{3}$; e Fcasca = fator de casca.

Os estoques de volume, biomassa e carbono e o sequestro de dióxido de carbono, por espécie coletada em 2007, foram calculados pelo emprego das seguintes expressões:

$V F c c_{i}=\left(\sum_{j=1}^{n_{i}} v f c c_{i j}\right) / A_{k} ; V F s c_{i j}=\left(\sum_{j=1}^{n_{j}} v f s c_{i j}\right) / A_{k} ; V F C_{i}=$ $\left(\sum_{j=1}^{n_{i}} v f c_{i j}\right) / A_{k} ; V T c c_{i}=\left(\sum_{j=1}^{n_{i}} v t c c_{i j}\right) / A_{k}$

$V G c c_{i}=\left(\sum_{j=1}^{n_{i}} v g c c_{i j}\right) / A_{k} ; V G c_{i}=\left(\sum_{j=1}^{n_{i}} v g c_{i j}\right) / A_{k} ; V G s c_{i}=$

$V G c c_{i}-V G c_{i}$

BFuste $=D B m \cdot V F s c+D B c \cdot V F c ; B G a l h o=D B m$.

$(V G c c-V G c)+D B c \cdot V G c ;$ BTotal = BFuste + BGalho

CTotal $=0,50 \cdot$ BTotal; e $C_{2}$ Total $=3,67 \cdot$ CTotal

em que $V F c c=$ volume dos fustes com casca, em $\mathrm{m}^{3} \cdot \mathrm{ha}^{-1} ; V F s c=$ volume dos fustes sem casca, em $\mathrm{m}^{3} \cdot \mathrm{ha}^{-1} ; V F_{C}=$ volume de casca dos fustes, em $\mathrm{m}^{3} \cdot \mathrm{ha}^{-1}$; $V T c c=$ volume total com casca, em $\mathrm{m}^{3} \cdot \mathrm{ha}^{-1} ; V G C c=$ volume dos galhos com casca, $\mathrm{em} \mathrm{m}^{3} \cdot \mathrm{ha}^{-1} ; V G c=$ volume de casca dos galhos, em $\mathrm{m}^{3} \cdot \mathrm{ha}^{-1} ; \mathrm{A}_{\mathrm{k}}=$ área da amostragem, em hectare, respectivamente, 0,80 ha para a Mata $1(k=1)$ e 0,30 ha para a Mata $2(\mathrm{k}=2) ; D B m=$ densidade básica da madeira, em t.m ${ }^{-3} ; D B c=$ densidade básica da casca, em t. $\mathrm{m}^{-3} ; V G C c=$ volume dos galhos com casca, em $\mathrm{m}^{3} \cdot \mathrm{ha}^{-1} ;$ VGsc $=$ volume dos galhos sem casca, em $\mathrm{m}^{3} \cdot \mathrm{ha}^{-1}$; VGc $=$ volume de casca dos galhos, em $\mathrm{m}^{3} \cdot \mathrm{ha}^{-1} ;$ BFuste $=$ biomassa de fuste com casca, em tB.ha ${ }^{-1}$; BGalho = biomassa de galho, em tB.ha-1; BTotal = biomassa total com casca, em tB.ha' ${ }^{-1}$; CTotal = carbono total, em tC.ha ${ }^{-1}$; e $\mathrm{CO}_{2}$ Total $=$ dióxido de carbono total, em tCO $\mathrm{tCh}_{2} \cdot \mathrm{ha}^{-1}$.

Para calcular os estoques de biomassa das espécies não coletadas, consideraram-se as médias das densidades básicas da madeira $(0,65)$ e da casca $(0,49)$ iguais às médias das densidades básicas da madeira e da casca das espécies coletadas.

Brown et al. (1986) e Goudriaan (1992) consideraram que metade (50\%) da biomassa de matéria seca em estufa é composta por carbono. $O$ fator de conversão de estoque de carbono em sequestro de carbono igual a 3,67 foi obtido pela razão entre a massa molecular do dióxido $\left(\mathrm{CO}_{2}\right)$, igual a 44 e a massa atômica do carbono (C) igual a 12 (BROWN et al., 1986; HOEN; SOLBERG, 1994; ORTIZ, 1997; RAMÍREZ et al., 1997).

Foram totalizados, por espécies coletadas e não coletadas, respectivamente, os estoques de volumes totais com casca (VTcc) e de fustes com casca (VFcc) e sem casca (VFsc), biomassa, carbono e dióxido de carbono, em 2002 e em 2007. Esses resultados fundamentaram as estimativas de crescimentos periódicos anuais.

\section{RESULTADOS}

\subsection{Estrutura Fitossociológica}

Em 2007, no inventário das parcelas permanentes da Mata 1 o diâmetro médio foi de 10,8 cm e a altura total média de 8,5 m, com predominância (78\%) das alturas totais no estrato de 5 a $12 \mathrm{~m}$, o que remete à classificação de estágio médio de sucessão (BRASIL, 2007). O número de indivíduos por hectare variou de 1.040 a 2.240 n.ha-1 ${ }^{-1}$ com média de 1.459 n.ha ${ }^{-1}$ e desviopadrão de 359 n.ha-1 ; a dominância total variou de 7,55 a 29,33 $\mathrm{m}^{2}$.ha- ${ }^{-1}$, com média de $16,82 \mathrm{~m}^{2}$.ha ${ }^{-1}$ e desvio-padrão de $5,54 \mathrm{~m}^{2}$.ha-1; e o volume total com casca oscilou de 39,74 a 275,07 $\mathrm{m}^{3} \cdot$ ha $^{-1}$, com média de $115,40 \mathrm{~m}^{3}$.ha ${ }^{-1} \mathrm{e}$ desvio-padrão de $66,47 \mathrm{~m}^{3} \cdot \mathrm{ha}^{-1}$.

Revista Árvore, Viçosa-MG, v.36, n.1, p.169-179, 2012 
Na Mata 2, em 2007, o diâmetro médio foi de $13,3 \mathrm{~cm}$ e a altura total média, de 10,4 m, com 65\% das alturas totais no estrato de 5 até $12 \mathrm{~m}$ e 35\% no estrato de alturas totais iguais ou superiores a 12 m, o que remete à classificação de estágio médioavançado de sucessão (BRASIL, 2007). O número de indivíduos por hectare variou de 1.340 a 2.180 n.ha-1, com média de 1.897 n.ha-1 e desvio-padrão de 318 n.ha- ${ }^{-1}$; dominância total de 16,94 a 35,70 m².ha-1, com média de $25,73 \mathrm{~m}^{2}$.hä ${ }^{-1}$ e desvio-padrão de 7,06 $\mathrm{m}^{2}$.ha ${ }^{-1}$; e volume total com casca, que oscilou de 135,6 a 392,07 m³.ha-1, com média de $245,20 \mathrm{~m}^{3}$.ha-1 e desvio- padrão de 99,66 m³ $\cdot \mathrm{ha}^{-1}$.

Verifica-se, na Tabela 1, que na Mata 1, no inventário realizado em 2002, as 34 (29\%) espécies do estrato arbóreo (DAP e” 5,0 cm) selecionadas para coletas de madeira e casca para determinação de densidades básicas contribuíram com 71,0\% do VI\% e volumes totais com casca de 83,06 m³.ha-1 (76,9\%) e 92,11 m³ ha $^{-1}$ (79,8\%), respectivamente, nos inventários de 2002 e 2007 . As demais 83 (71\%) espécies não coletadas contribuíram, em 2002, com 23,1\% e, em 2007, com 20,2\% do volume total com casca da Mata 1.

Na Mata 2, nos inventários realizados em 2002 e 2007, verifica-se, na Tabela 2, que 16 (18,0\%) espécies coletadas contribuíram com 55,6\% do VI\% e volume total com casca de $188,51 \mathrm{~m}^{3}$.ha-1 $(83,3 \%)$ e 202,4 m³ ha $^{-1}$ (82,5\%).

Nota-se, nessa tabela, que em 2007 as espécies do estrato arbóreo (DAP $\geq 5,0 \mathrm{~cm}$ ) com os maiores valores de importância fitossociológica (VI\%) foram: na Mata 1 (Tabela 1), Xylopia sericea, Mabea fistulifera, Apuleia leiocarpa, Ocotea lanata, Diatenopteryx sorbifolia, Brosimum guianense, Cupania oblongifolia, Pouteria torta, Lecythis lurida, Astronium fraxinifolium e Cordia sellowiana; e na Mata 2 (Tabela 2): Pseudopiptadenia contorta, Mabea fistulifera, Brosimum guianense, Apuleia leiocarpa, Pouteria torta, Cupania oblongifolia, Ocotea lanata, Myrcia fallax, Tapirira guianensis, Ocotea odorifera, Rollinia sylvatica e Lecythis lurida.

\subsection{Estoque e crescimento em volume, biomassa, carbono e dióxido de carbono}

As populações de maiores estoques de volume total com casca $\left(\mathrm{m}^{3} \cdot \mathrm{ha}^{-1}\right)$, em 2007, foram: na Mata 1: Mabea fistulifera $(15,89)$, Xylopia sericea $(15,79)$ e Apuleia leiocarpa (13,35), totalizando 45,03 $\mathrm{m}^{3} \cdot \mathrm{ha}^{-1}$ (39,0\%); e na Mata 2, Pseudopiptadenia contorta $(94,80)$, Mabea fistulifera $(25,98)$, Apuleia leiocarpa $(12,89)$, Brosimum guianense $(11,97)$, Tapirira guianensis $(11,14)$ e Cupania oblongifolia $(11,00)$, perfazendo $167,78 \mathrm{~m}^{3} \cdot \mathrm{ha}^{-1}(68,4 \%)$.

A mata em estágio médio de sucessão secundária (Tabela 1) apresentou crescimentos de 1,49 m ha $^{-1} \cdot$ ano $^{-1}$, 0,27 tB.ha-1 ano $^{-1}$, 0,13 tC.ha- . ano $^{-1} \mathrm{e}$ $0,50 \mathrm{tCO}_{2} \cdot \mathrm{ha}^{-1}$.ano ${ }^{-1}$. Já a mata em estágio médio avançado de sucessão da vegetação secundária (Tabela 2) mostrou

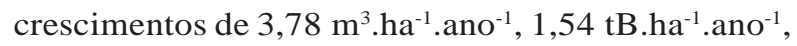

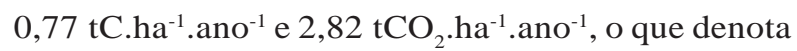
taxas de crescimento relativo do estoque de dióxido de carbono de $0,37 \%$ na área em estágio médio de sucessão e 1,05\% na área em estágio médio avançado. Em termos absolutos, a mata em estágio médio avançado sequestrou, no período de 2002 a 2007, mais de cinco vezes e meia dióxido de carbono do que a mata em estágio médio.

A dinâmica do estoque de carbono relaciona-se com o estágio de sucessão da floresta e com o grupo ecológico das espécies. Na área em estágio médio (Tabela 1), as espécies com maiores crescimentos foram: Xylopia sericea (SI), com 4,10 tCO .ha $^{-1}$. ano ${ }^{-1}$; Diatenopteryx sorbifolia (ST), com 1,63 tCO .ha $^{-1}$. ano $^{-1}$; Mabea fistulifera (P), com 1,57 $\mathrm{tCO}_{2} \cdot \mathrm{ha}^{-1}$.ano-1; e Ocotea lanata (SI), com 0,95 $\mathrm{tCO}_{2} \cdot \mathrm{ha}^{-1} \cdot$ ano $^{-1}$. Apuleia leiocarpa (SI), Rollinia sylvatica (SI), Myrcia fallax (SI), Inga stipularis (SI), Cecropia pachystachya (PI), Matayba elaeagnoides (SI) e Solanum swartzianum (P) acumularam reduções de $0,55 \mathrm{tCO}_{2} \cdot \mathrm{ha}^{-1} \cdot \mathrm{ano}^{-1}$. Na área em estágio médio avançado (Tabela 2), as espécies com maiores crescimentos foram: Pseudopiptadenia contorta (SI), com 6,04 tCO .ha $^{-1}$. ano ${ }^{-1}$; Brosimum guianense $(\mathrm{C})$, com 1,56 tCO $\mathrm{tha}^{-1} \cdot \mathrm{ano}^{-1}$; Cupania oblongifolia (SI), com 1,21 tCO $2 \cdot \mathrm{ha}^{-1} \cdot \mathrm{ano}^{-1}$; Ocotea odorifera (SI), com 1,09 tCO $\mathrm{tCh}^{-1} \cdot \mathrm{ano}^{-1}$; e Pouteria torta (SI), com 1,01 tCO $\cdot \mathrm{ha}^{-1} \cdot \mathrm{ano}^{-1}$. Mabea fistulifera (P), Tapirira guianensis (P), Machaerium triste (SI), Connarus sp. (SC) e Cordia sellowiana (P) apresentaram, no período de 2002 a 2007, balanço negativo de biomassa e carbono. Destaque para Mabea fistulifera (P), que apresentou balanço positivo nos estágios médio e negativo do estágio médio avançado. 


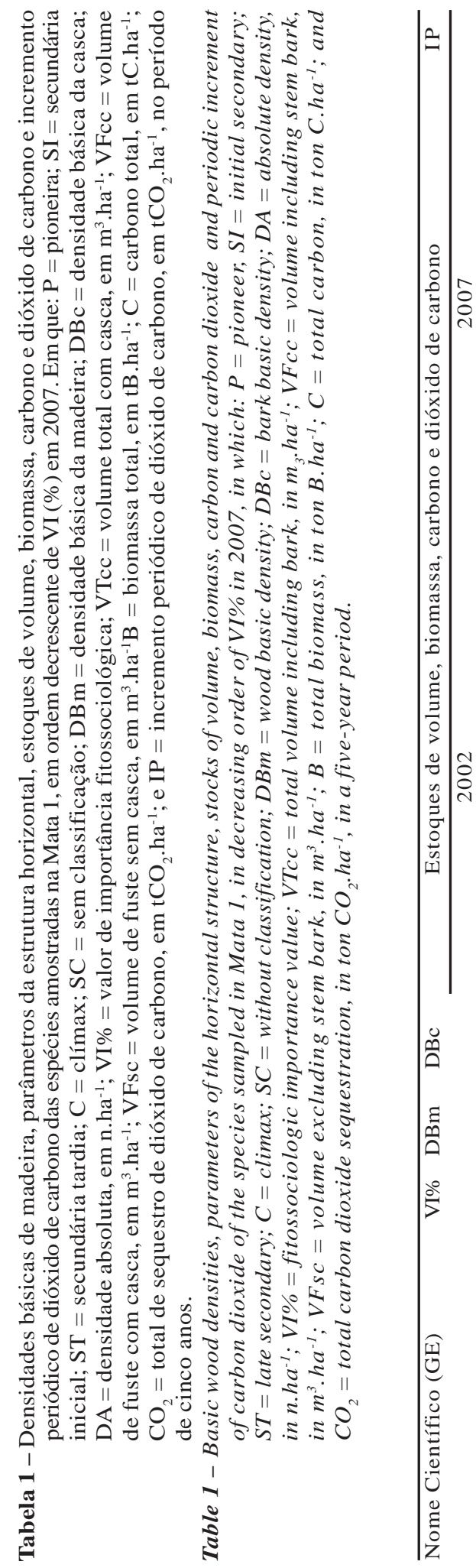

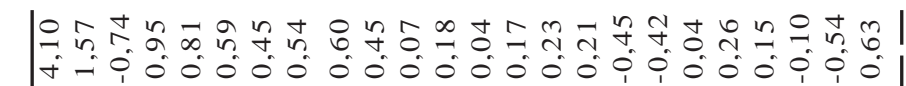

出

चै

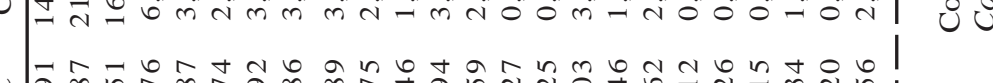

U

๓

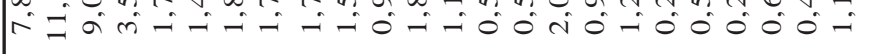

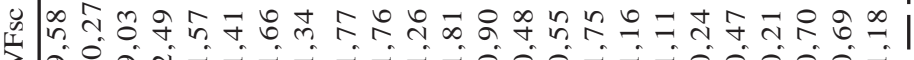

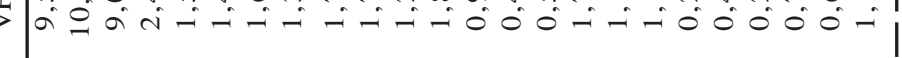

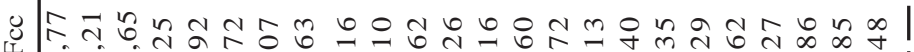

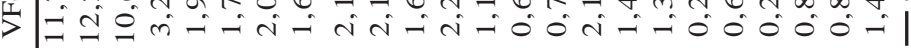

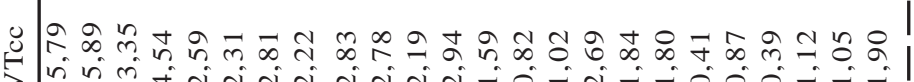

मून

|N

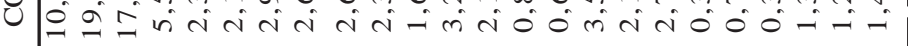

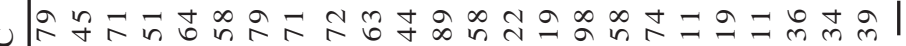

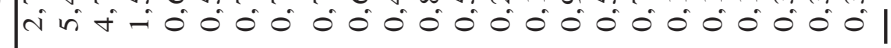

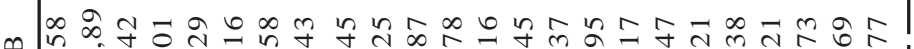

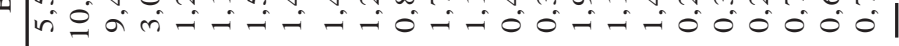

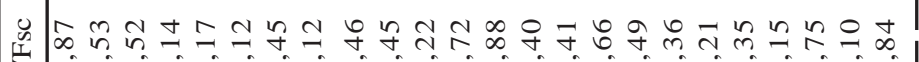

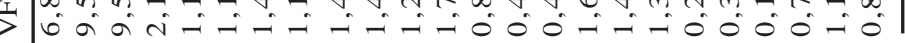

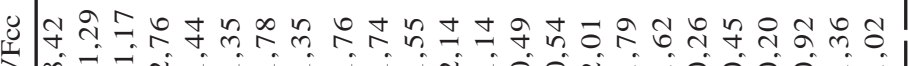

$>$

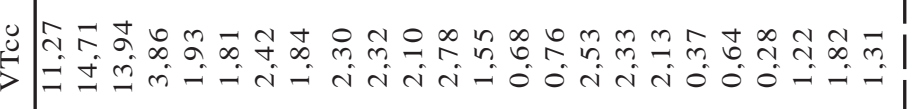

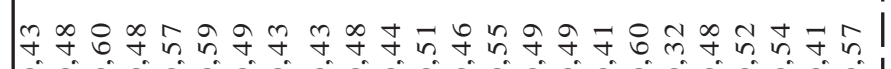

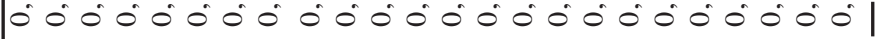

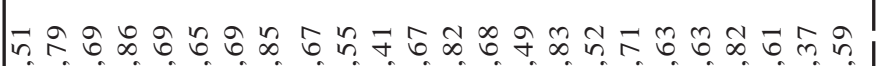

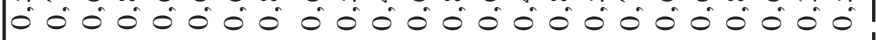

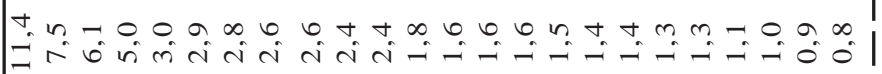

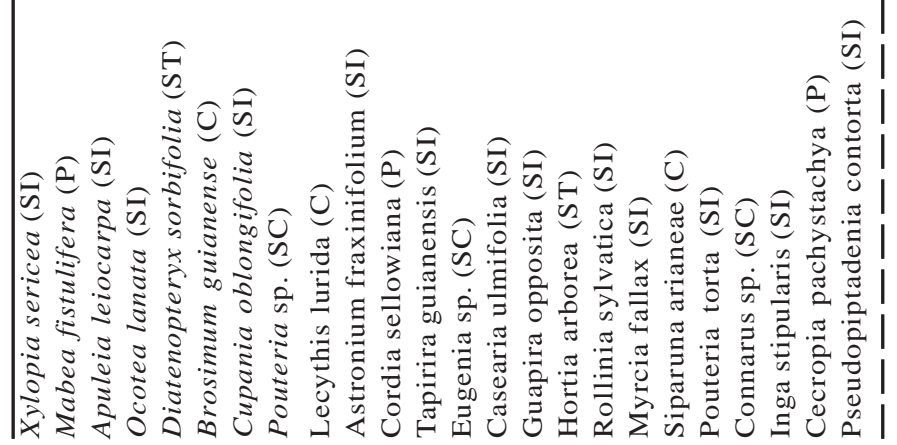





Revista Árvore, Viçosa-MG, v.36, n.1, p.169-179, 2012 
|ำ

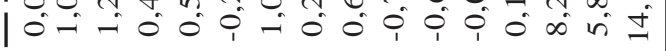

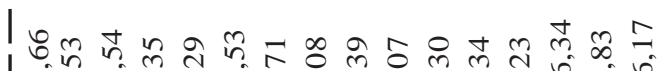

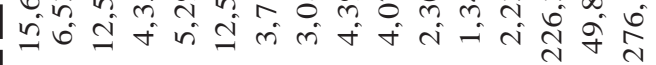

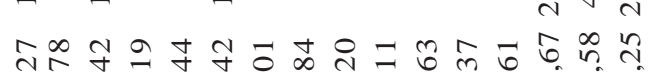

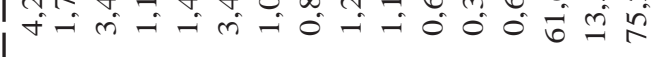
|

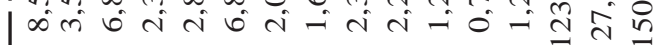

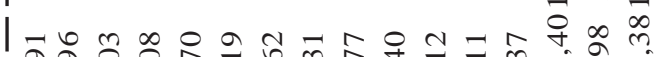

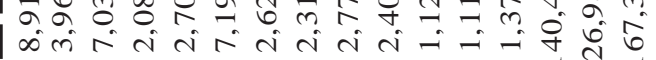
约№는

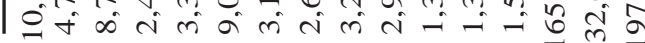
| ปैं | ఫึ๊

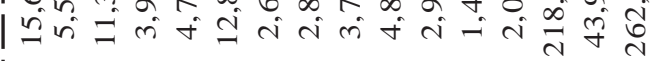

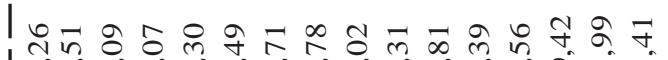

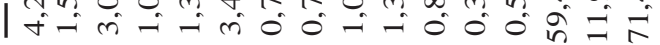
|

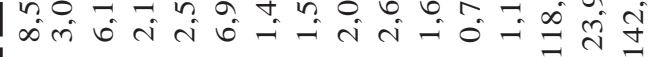
| किली

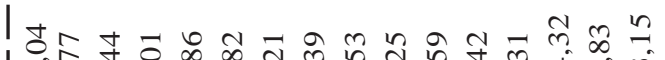
वें 1

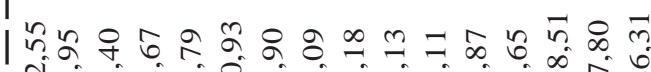

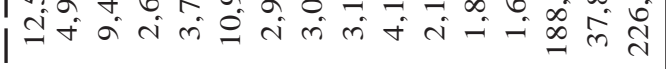
|

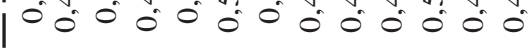
|

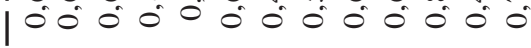
I $m$ m

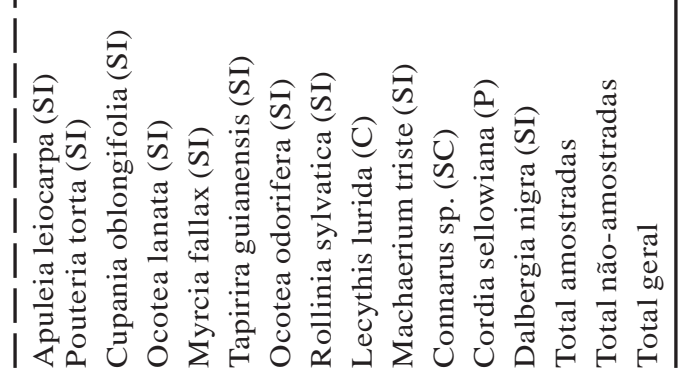

\section{DISCUSSÃO}

\subsection{Estrutura Fitossociológica}

Mesmo entre áreas de Florestas Estacionais

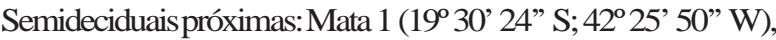

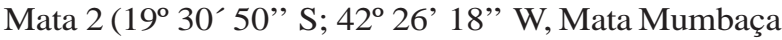
(19 44' 18" S e 36 28' 48”' W)(DRUMOND et al., 1996)), MataSalão Dourado (19 $29^{\prime} 24^{\prime \prime}-19^{\circ} 48^{\prime} 18^{\prime \prime}$ 'Se $42^{\circ} 28^{\prime} 18^{\prime \prime}$ $42^{\circ} 38^{\prime} 30^{\prime \prime}$ W) (DRUMOND et al., 1996) e PFERD-Parque Estadual do Rio Doce, MG $\left(19^{\circ} 29^{\prime}\right.$ - $19^{\circ} 48^{\prime} \mathrm{S}$ e $42^{\circ} 28^{\prime}$ $42^{\circ} 38^{\prime}$ W) (LOPES et al., 2002), observaram-se grandes variabilidades florística e estrutural. Pelos resultados dos estudos de Drumond et al. (1996), a Mata Mumbaça apresentou, no levantamento de 1994, 23 famílias pertencentes a 34 gêneros e 43 espécies, $H^{\prime}$ igual a 3,09, densidade total de $1.247 \mathrm{n} \cdot \mathrm{ha}^{-1}$, dominância total de $16,70 \mathrm{~m}^{2}$.ha ${ }^{-1}$ e volume total com casca de 205,30 $\mathrm{m}^{3}$.ha ${ }^{-1}$. Em Souza (2008), a Mata Mumbaça apresentou: na "faixa rampa baixa”, 32 famílias, 72 gêneros, 93 espécies, H' igual a 3,64, DTA de 735 n.ha-1 e DoT de 24,60 m².ha-1 ; na "faixa baixa encosta côncava”, 32 família, 64 gêneros, 89 espécies, $\mathrm{H}^{\prime}$ igual a 3,49, DTA de 820 n.ha ${ }^{-1}$ e DoT de $18,46 \mathrm{~m}^{2}$.ha-1; na "faixa alta encosta côncava", 31 famílias, 66 gêneros, 89 espécies, H' igual a 3,55, DTA de 870 n.ha ${ }^{-1}$ e DoT de 17,60 $\mathrm{m}^{2}$.ha-1; ; e na "faixa topo", 34 famílias, 66 gêneros, 88 espécies, H’ igual a 3,73, DTA de 822 n.ha ${ }^{-1}$ e DoT de 19,36 m².ha-1. A Mata Salão Dourado (DRUMOND et al., 1996) apresentou, no levantamento de 1994, 38 famílias pertencentes a 80 gêneros e 114 espécies, $\mathrm{H}^{\prime}$ igual a 3,85, densidade total de 1.690 n.ha-1 ${ }^{-1}$ dominância total de 30,65 m².ha-1 e volume total com casca de $381,76 \mathrm{~m}^{3}$.ha-1. . O trecho do PFERD MG (LOPES et al., 2002), avaliado numa amostragem por pontos quadrantes, apresentou 40 famílias pertencentes a 110 gêneros e 144 espécies, com H' de 3,98, densidade total de 1.569 n.ha ${ }^{-1}$ e dominância total de $26,94 \mathrm{~m}^{2}$.ha- ${ }^{-1}$.

As espécies Mabea fistulifera, Apuleia leiocarpa, Pouteria torta, Brosimum guianense e Pseudopiptadenia contorta figuram entre as 10 espécies de maiores VI\% da Mata 2 e da Mata Mumbaça (DRUMOND et al., 1996). A espécie Pseudopiptadenia contorta consta como a espécie de maior VI\% na Mata 2 e nas Matas Salão Dourado e Mumbaça (DRUMOND et al., 1996) e a quinta de maior VI\% no PFERD (LOPES et al., 2002). No ano de 2007, na Mata Mumbaça (SOUZA, 2008) Pseudopiptadenia contorta foi relacionada como a terceira de maior 
VI\% na "faixa topo" e na "faixa baixa encosta”, a sexta na "faixa rampa baixa" e a nona na "faixa alta encosta côncava”.

As espécies Apuleia leiocarpa, Pseudopiptadenia contorta, Matayba elaeagnoides, Ocotea odorifera e Tapirira guianensis constam da lista das espécies de maior VI\% do trabalho de Meira Neto et al. (1997a); Apuleia leiocarpa, Lecythis lurida, Dalbergia nigra e Pseudopiptadenia contorta constam entre as 10 de maior VI\% do estudo de Meira Neto et al. (1997b); e Ocotea odorifera foi a espécie de maior VI no trabalho de Meira Neto et al. (1997c).

\subsection{Estoques e crescimento em volume, biomassa, carbono e dióxido de carbono}

As estimativas médias de estoques de biomassa e carbono da área em estágio médio de sucessão da vegetação secundária (mata 1) de 73,09 tB.ha ${ }^{-1}$ e 36,54 tC.ha- ${ }^{-1}$ e da área em estágio médio avançado (mata 2) de 150,50 tB.ha-1 e 75,25 tC.ha-1 superaram as médias obtidas por Souza-Lima et al. (2003) e Ribeiro et al. (2010) em Mata Atlântica em estágio de sucessão secundária na Zona da Mata, MG. SouzaLima et al. (2003) estimaram os estoques de biomassa e carbono de uma floresta secundária de cerca de 37 anos de idade de regeneração natural, em 65 tB.ha-1 e 29 tC.ha-1. Já Ribeiro et al. (2010) obtiveram para o componente arbóreo (DAP e” 5,0 cm) de uma Floresta Estacional Semidecidual em estágio médio de sucessão, em Viçosa, MG, 38,99 tB.ha-1 e 19,50 \pm 8,08 tC.ha-1 . Entretanto, os estoques da Mata 1 e da Mata 2 foram inferiores aos estoques de biomassa (166,67 tB.ha $\left.{ }^{-1}\right)$ e carbono (83,34 tC.ha-1) de fuste sem casca de Floresta Estacional Semidecidual Montana madura (estágio secundário avançado clímax), em Viçosa, MG (RIBEIRO et al., 2009). Em estudos realizados em Floresta Atlântica, em Antonina, PR, Tiepolo et al. (2002) citaram que a tipologia submontana foi a que apresentou maiores estoques de carbono, com 135,89 tC.ha-1, e os demais estágios de regeneração exibiram as seguintes cifras: 106,19 tC ha-1 (estágio médio avançado), 101,96 tC ha-1 (estágio médio) e 42,89 tC.ha-1 (estágio inicial). Cunha et al. (2009) obtiveram, para o compartimento arbóreo (DAP $\geq 10,0 \mathrm{~cm}$ ) de dois fragmentos de Floresta Ombrófila Densa Montana com cerca de 40 anos de idade de regeneração da vegetação secundária, Município de Santa Madalena, RJ, estoques de 65,9 e 68,4 tC.ha-1 na parte aérea.

Revista Árvore, Viçosa-MG, v.36, n.1, p.169-179, 2012
As tendências de evolução do estoque e crescimentos foram coerentes com a dinâmica de sucessão florestal, ou seja, após o ponto de inflexão da curva de produção, quanto maior o estoque de biomassa vegetal viva do componente arbóreo, menor o crescimento (ODUM, 1986; BICK et al., 998). Estudos sobre estoques de carbono em tipologias de Floresta Atlântica são mais frequentes (VIEIRA et al., 2008), ao passo que os estudos de dinâmica ainda são escassos (BOINA, 2008). No bioma Floresta Amazônica já há estudos analisando estoques e dinâmica de carbono em florestas primárias (PHILIPS et al., 1998; HIGUCHI et al., 2004) e secundárias (SALOMÃO et al., 1998). Em florestas secundárias de 5, 10 e 20 anos de regeneração natural, Salomão et al. (1998) estimaram estoques de 13, 44 e 82 tB.ha-1, com incremento médio

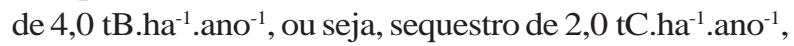
que equivale a $5,34 \mathrm{tCO}_{2} \cdot$ ha $^{-1}$. ano $^{-1}$. Em florestas primárias na região de Manaus, AM, Higuchi et al. (2004) obtiveram estimativa de 1,2 tC.ha ${ }^{-1} \cdot$ ano $^{-1}$.

\section{CONCLUSÃO}

A mata em estágio médio avançado de sucessão da vegetação secundária estocou, no período de 2002 a 2007, mais de cinco vezes e meia dióxido de carbono do que a mata em estágio médio, porém apresentou menor crescimento ou capacidade de sequestrar carbono.

A estocagem e o sequestro de dióxido de carbono estão relacionados com a composição florística, estrutura fitossociológica e estágio sucessional da floresta nativa. Na mata em estágio médio, as espécies com maiores valores de importância e crescimentos de volume, biomassa, carbono e dióxido de carbono foram Xylopia sericea, Diatenopteryx sorbifolia, Mabea fistulifera e Ocotea lanata. E as espécies com os maiores estoques foram Xylopia sericea, Mabea fistulifera, Apuleia leiocarpa e Ocotea lanata. Na mata em estágio médio avançado, as espécies com maiores valores de importância e crescimentos de volume, biomassa, carbono e dióxido de carbono foram Pseudopiptadenia contorta, Brosimum guianense, Cupania oblongifolia, Ocotea odorifera e Pouteria torta. E as espécies com os maiores estoques de $\mathrm{CO}_{2}$ foram Pseudopiptadenia contorta, Mabea fistulifera, Apuleia leiocarpa, Brosimum guianense, Cupania oblongifolia e Tapirira guianense. O destaque foi para Mabea fistulifera, que apresentou balanço positivo de carbono no estágio médio e negativo no estágio médio avançado. 


\section{REFERÊNCIAS}

ASSOCIAÇÃO BRASILEIRA DE NORMAS TÉCNICAS - ABNT. NBR 7190: Projetos de estruturas da madeira. Rio de Janeiro: 1997. 107p.

BARNETT, J. R.; JERONIMIDIS, G. Wood quality and its biological basis. Oxford: CRC Press, 2003. 223p.

BICK, U. et al. Assessment and measurement of forestry key parameters for the evaluation of tropical forest management. Plant Research and Development, v.47/48, p.38-61, 1998.

BOINA, A. Quantificação de estoques de biomassa e de carbono em Floresta Estacional Semidecidual, vale do rio doce, Minas Gerais. 2008. 89f. Dissertação (Mestrado em Ciência Florestal) - Universidade Federal de Viçosa, Viçosa, MG, 2008.

BRASIL. Resolução/CONAMA № 392, de 25 de junho de 2007. Define vegetação primária e secundária de regeneração de Mata Atlântica no Estado de Minas Gerais. Diário Oficial da República Federativa do Brasil. Brasília, 26 de junho de 2007.

BRASIL. Ministério da Ciência e Tecnologia. Emissões e remoções de dióxido de carbono devidas a mudanças nos estoques de florestas plantadas. Primeiro Inventário Brasileiro de Emissões Antrópicas de Gases de Efeito Estufa - Relatórios de Referência. 2006. Disponível em: <http://www.mct.gov.br/ index.php/content//view/21456.html >. Acesso em: 23 jan. de 2008.

BROWER, J. E.; ZAR, J. H. Field and laboratory methods for general ecology. 2.ed. Iowa: Wm. C. Brown, 1984. 22p.

BROWN, S.; LUGO, A.; CHAPMAN, J. Biomass of tropical tree plantations and its implications for the global carbon budget. Canadian Journal of Forestry Research, v.16, p.390-394, 1986.

CHAVES, J. et al. Tree alometriy and improved estimation of carbon stocks and balance in tropical forests Oecologia, v.145, n.1, p.87-99, 2005.
CUNHA, G. M. et al. Biomassa e estoque de carbono e nutrientes em florestas montanas da Mata Atlântica na região norte do Estado do Rio de Janeiro. Revista Brasileira de Ciência do Solo, v.33, n.5, p.1175-1185, 2009.

DRUMOND, M. A. et al. Alterações fitossociológicas e edáficas na Mata Atlântica em função das modificações da cobertura vegetal. Revista Árvore, v.20, n.4, p.451466, 1996.

FOODY, G. M. et al. Identifying terrestrial carbon sinks: classification of successional stages in regenerating tropical forest from landsat TM data. Remote Sense Environment, v.55, p.205-216. 1996.

\section{GLOBAL FOREST RESOURCES ASSESSMENT}

2010 (FRA, 2010). Rome: FAO, 2010. 371p.

Disponível em: www.fao.org/forestry/fra/ fra2010/en/

GOUDRIAAN, J. Adónde va el gás carbônico?: El papel de La vegetación. Mundo Científico, v.126, n.12, p.687-692, 1992.

HIGUCHI, N. et al. Dinâmica e balanço do carbono da vegetação primária da Amazônia Central. Floresta, v.34, n.3; p.295-304, 2004.

HOEN, H.; SOLBERG, B. Potencial and economic efficiency of cabon sequestration in Forest biomass through silvicultural management. Forest Science, v.40, n.3, p.429-451, 1994.

FUNDAÇÃO INSTITUTO BRASILEIRO DE GEOGRAFIA E ESTATÍSTICA - IBGE. Mapa de vegetação do Brasil. Rio de Janeiro: 2004.

LAL, R.; KIMBLE, J.; STEWART, B. A. World soils as a source or sink for radiatively-active gases. In: LAL, R. et al. (Ed.). Soil management and greenhouse effect. Boca Raton: CRC Lewis Publishers, 1995. p.1-7.

LOPES, W. P. et al. Estrutura fitossociológica de um trecho de vegetação arbórea no Parque Estadual do Rio Doce - Minas Gerais, Brasil. Acta Botânica Brasílica, v.16, n.4, p.443-456, 2002.

Revista Árvore, Viçosa-MG, v.36, n.1, p.169-179, 2012 
MEIRA NETO, J. A. et al. Estrutura de uma Floresta Estacional Semidecidual insular em área diretamente afetada pela Usina Hidrelétrica de Pilar, Ponte Nova, Zona da Mata de Minas Gerais. Viçosa, Revista Árvore, v.21, n.4, p.493-500, 1997a.

MEIRA NETO, J. A. et al. Estrutura de uma Floresta Estacional Semidecidual Submontana em área diretamente afetada pela Usina Hidrelétrica de Pilar, Ponte Nova, Zona da Mata de Minas Gerais. Viçosa, Revista Árvore, v.21, n.3, p.337-344, 1997b.

MEIRA NETO, J. A. et al. Estrutura de uma Floresta Estacional Semidecidual Aluvial em área diretamente afetada pela Usina Hidrelétrica de Pilar, Ponte Nova, Zona da Mata de Minas Gerais. Viçosa, Revista Árvore, v.21, n.2, p.213-219, 1997c.

MUELLER-DOMBOIS, D., ELLENBERG, H. Aims and methods of vegetation ecology. New York: John Wiley \& Sons, 1974. 547p.

MULLER-LANDAU, H. C. Interespecific and inter-site variation in wood specific gravity of tropical trees. Biotropica, v.36, n.1, p.20-32, 2004.

MYERS, N. et al. Biodiversity hotspots for conservation priorities. Nature, v.403, n.1, p.852-858, 2000.

ODUM, E. P. Ecologia. Rio de Janeiro: Guanabara, 1986. 434p.

ORTIZ, R. Costa Rican secondary Forest: an economic option for joint implementation initiatives to reduce atmospheric $\mathbf{C O}_{2}$. Draft paper presented for inclusion in the Beijer Seminar in Punta Leona. Costa Rica: 1997. 19p.

PASSOS, C. A. M.; BRAZ, E. M. Manejo florestal e silvicultura de precisão na Amazônia. Revista da Madeira, n. 85, nov. 2004. Disponível em: $<$ http://www.remade.com.br/pt/ revista_materia.php?edicao $=85$ eid $=651>$. Acesso em: 25 jan. 2008.

PHILLIPS, O. L. et al. Changes in the carbon balance of tropical forests: evidence from longterm plots. Science, v.282, p.439-442, 1998.
RAMÍREZ, O.; GÓMEZ, M.; SHULTZ, S. Valuing the contribution of plantation forestry to the national accounts of Costa Rica from the ecological economics perspective. Costa Rica: Beijer Research Seminar, 1997. 28p.

RIBEIRO, S. C. et al. Quantificação de biomassa e estimativa de estoque de carbono em uma floresta madura no município de Viçosa, Minas Gerais. Revista Árvore, v.33, n.5, p.917-926, 2009.

RIBEIRO, S. C. et al. Quantificação de biomassa e estimativa de estoque de carbono em uma capoeira da Zona da Mata Mineira. Revista Árvore, v.34, n.3, p.495-504, 2010.

SALOMÃO, R.P.; NEPSTAD, D.C.; VIEIRA, I.C. Biomassa e estoque de florestas tropicais primária e secundária. In: (GASCON, C. MOUTINHO, P. (Ed.) Floresta Amazônica: Dinâmica, regeneração e manejo. LOCAL: EDITORA, 1998. p. $99-119$.

SILVA, C. A.; MACHADO, P. L. O. A. Seqüestro e emissão de carbono em ecossistemas agrícolas: Estratégias para o aumento dos estoques de matéria orgânica em solos tropicais. Rio de Janeiro: Embrapa Solos, 2000. 23p. (Documentos, 19).

SOARES, C. P. B.; PAULA NETO, F.; SOUZA, A. L. Dendrometria e inventário florestal. Viçosa, MG: Universidade Federal de Viçosa, 2006. 276p.

SOUSA LIMA, J. A. et al. Estimativas de biomassa e estoques de carbono de uma floresta secundária em Minas Gerais Rio de Janeiro: Embrapa Solos, 2003. 18p. (Boletim de Pesquisa e Desenvolvimento, 20).

SOUZA, P. B. Diversidade florística e atributos pedológicos ao longo de uma encosta com floresta estacional semidecidual submontana, zona de amortecimento do Parque Estadual do Rio Doce, MG. 2008. 138f. Tese (Doutorado em Ciência Florestal) - Universidade Federal de Viçosa, Viçosa, MG, 2008. 
SWAINE, M. D.; WHITMORE, T.C. On the definition of ecological species groups in tropical rain forests. Vegetatio, v.75, n.1-2, p.81-86, 1988.

TIEPOLO, G.; CALMON, M.; FERRETTI, A. R. Measuring and monitoring carbon stocks at the guaraqueçaba climate action project, Paraná, Brazil. In: TAIWAN FORESTRY RESEARCH INSTITUTE. International Symposium on Forest Carbon Sequestration and Monitoring. 2002. p.98115. (Extension Series, 153)

VELOSO, H. P.; RANGEL FILHO, A. L. R.; LIMA, J. C. A. Classificação da vegetação brasileira, adaptada a um sistema universal. Rio de Janeiro: Instituto Brasileiro de Geografia e Estatística, 1991. 123p.
VIEIRA, S. A. et al. Estimation of biomass and carbon stocks: the case of the Atlantic Forest. Biota Neotropica, v.8, n.2, p.21-29, 2008.

VITAL, B. R. Métodos de determinação da densidade da madeira. Viçosa, MG: 1984. 21p. (Sociedade de Investigações Florestais, 1)

WATZLAWICK, L. F. et al. Fixação de carbono em Floresta Ombrófila Mista em diferentes estágios de regeneração. In: SANQUETA, C. R. et al. As florestas e o carbono. Curitiba: Universidade Federal do Paraná, 2002. Parte 8. p.153-173. 